\title{
Deeply Virtual Compton Scattering and Exclusive Meson Production
}

\author{
Nicole d'Hose ${ }^{1, \text { a }}$ \\ ${ }^{1}$ CEA-Saclay, Irfu/SPhN, 91191 Gif-sur-Yvette, France
}

\begin{abstract}
A selection of hard exclusive measurements (deeply virtual Compton Scattering and meson production) from past, present and future experimental programs are reviewed. The goal is the determination of the Generalized Parton Distributions which offer one of the most complete description of the partonic structure of the nucleon.
\end{abstract}

\section{The Generalized Parton Distributions and Hard Exclusive Reactions}

One of the main open questions in the theory of strong interaction is to understand how the nucleon is built from quarks and gluons, the fundamental degrees of freedom in QCD. The most general information on the partonic structure of hadrons is contained in the generalized parton correlation functions (GPCFs) [1, 2] which parameterize the fully unintegrated, off diagonal quark-quark correlators for a given hadron. These functions have a direct connection to the so-called Wigner distributions - the quantum mechanical analogues of classical phase space distributions of the hadron-parton system. The GPCFs are 'mother distributions' of the generalized parton distributions (GPDs) and the transverse momentum dependent parton distributions (TMDs), which can be considered as different projections or limiting cases of GPCFs. These two types of parton distributions, GPDs and TMDs, provide a complementary 3-dimensional picture of the nucleon, either in a mixed position-momentum representation for the former or in a pure momentum space for the latter. Moreover they contain important information on the orbital motion of partons inside the nucleon. While TMDs can be measured in the semi-inclusive deep inelastic scattering (SIDIS) or the Drell-Yan process, GPDs appear in the QCD description of hard exclusive reactions such as deeply virtual Compton scattering (DVCS) or hard exclusive meson production (HEMP).

The GPDs [3-5] are universal distributions which embody both, form factors observed in elastic scattering and parton distribution functions measured in deep inelastic scattering (DIS). Exclusive reactions as deeply virtual Compton scattering (see Fig. 1) can be interpreted in terms of GPDs when the photon virtuality $Q^{2}$ is large and the four-momentum transfer $t$ between the initial and final nucleons is small. In this case the factorization into a hardscattering part (calculable in perturbative QCD) and a nonperturbative nucleon structure is valid [6]. The virtual

\footnotetext{
a e-mail: nicole.dhose@cea.fr
}

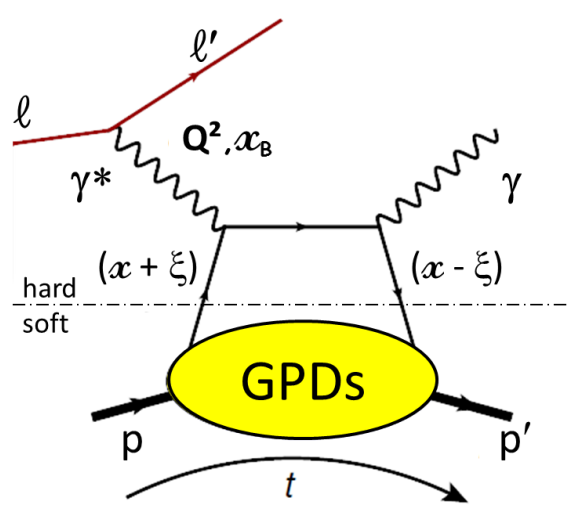

Figure 1. Feynman diagram of DVCS at leading order

photon exchanged in the lepton-nucleon scattering and defined by $Q^{2}$ and the Bjorken variable $x_{B}$, selects a quark in the nucleon with a longitudinal momentum fraction $x+\xi$ which is reabsorbed with a momentum fraction $x-\xi$ to leave the nucleon intact and to allow the emission of a real photon. The soft structure of the nucleon is described by GPDs which are function of $x, \xi, t, Q^{2}$. In DVCS or HEMP processes, the skewness $\xi$, which is half the difference between the initial and final longitudinal momentum fraction of the active partons, is related to the Bjorken variable $\xi=x_{B} /\left(2-x_{B}\right)$ in the Bjorken limit while the average value of the longitudinal momentum of the active parton $x$ is a mute variable which is integrated over. The transfer $t$ contains in addition to the longitudinal component a transverse one. This leads to information about the spatial transverse distribution of partons in addition to the longitudinal momentum distribution.

The DVCS final state is identical to that of the wellknown Bethe-Heitler (BH) process and hence both processes interfere on the level of amplitudes. This makes that hard exclusive single photon production is a powerful tool to extract DVCS amplitude which is at leading order in $\alpha_{S}$ and leading twist is a linear combination of Comp- 
ton form factors (CFF) linked to the GPDs. For the GPD $H$, the CFF can be written as:

$$
\begin{aligned}
\mathcal{H} & \sim \int_{-1}^{1} d x \frac{H(x, \xi, t)}{x-\xi+i \epsilon} \sim \mathfrak{R e} \mathcal{H}+i \mathfrak{I m} \mathcal{H} \\
& \sim \mathcal{P} \int_{-1}^{1} d x\left(\frac{H(x, \xi, t)}{x-\xi}\right)-i \pi H(\xi, \xi, t)
\end{aligned}
$$

The imaginary part of the CFF allows the direct determination of the GPD at the specific values $x= \pm \xi$ while the real part is a convolution of the $x$-dependence of the GPD with the kernel $1 /(x-\xi)$. There exists a dispersion relation which relates the real and imaginary parts to the Dterm which can be interpreted in the Breit frame in terms of shear forces and radial distribution of pressure inside the nucleon [7] in order to describe confinement.

$$
\mathfrak{R} \mathcal{H}(\xi, t)=\mathcal{P} \int_{-1}^{1} d x \frac{\mathfrak{J} \mathrm{m} \mathcal{H}(x, t)}{x-\xi}+D(t)
$$

The real and imaginary parts can be accesses separately through the interference between BH and DVCS.

They exist four chiral-even GPDs which conserve quark helicity, they are $H, \tilde{H}, E, \tilde{E}$ and four chiral-odd GPDs. Each GPD has a partner in the TMD sector. The GPDs $H^{f}$ and $\tilde{H}^{f}$ (f can be any quark flavor u,d,s or gluon g) describe the case of nucleon helicity conservation and include in the forward limit $\xi=0$ and $t=0$ the wellmeasured parton density $q^{f}(x)$ and the helicity distribution $\Delta q^{f}(x)$ respectively. The GPDs $E^{f}$ and $\tilde{E}^{f}$ have no such analogue. They allow for the possibility of the nucleon helicity flip. As the nucleon changes helicity but the massless quark does not, the angular momentum conservation implies a transfer of orbital angular momentum. This is epitomized in the Ji sum rule [5] which establishes the relation between the total angular momentum of a given parton species $\mathrm{f}$ and the forward limit of the $2^{\text {nd }}$ moment in $x$ of the sum of the GPDs $H^{f}$ and $E^{f}$.

$$
\begin{aligned}
J_{f} & =1 / 2 \lim _{t \rightarrow 0} \int_{-1}^{1} d x x\left(H^{f}(x, \xi, t)+E^{f}(x, \xi, t)\right) \\
1 / 2 & =\sum_{q=u, d, s} J^{q}+J^{g}
\end{aligned}
$$

The GPD $E$, related to orbital angular momentum, has for partner the Sivers function. The first moment in $x$ of the GPDs are related to the nucleon form-factors. For example:

$$
\int_{-1}^{1} d x\left(H^{f}(x, \xi, t)=F_{1}^{f}(t) ; \quad \int_{-1}^{1} d x\left(E^{f}(x, \xi, t)=F_{2}^{f}(t)\right.\right.
$$

where $F_{1}^{f}$ and $F_{2}^{f}$ are the contributions to the Dirac and Pauli form factors from quark flavor $f$. A particularly simple physical interpretation for GPDs can be developed in the limiting case $\xi=0 . H^{f}\left(x, 0, t=-\Delta_{\perp}^{2}\right)$ is the Fourier transform of the probability density to find a parton $f$ with longitudinal momentum fraction $x$ with respect to its transverse distance $b_{\perp}$ from the centre of momentum of the nucleon (impact parameter representation) [8]:

$$
q^{f}\left(x, b_{\perp}\right)=\int \frac{d^{2} \Delta_{T}^{2}}{2 \pi^{2}} e^{-i \Delta_{\perp} \cdot b_{\perp}} H^{f}\left(x, 0, t=-\Delta_{\perp}^{2}\right)
$$

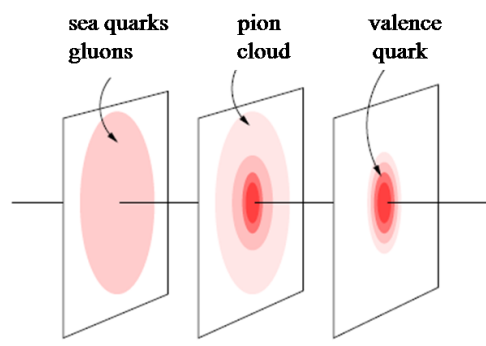

(a)

(b) $x \sim 0.003 \quad x \sim 0.03 \quad x \sim 0.3$

Figure 2. Nucleon tomography: sketch of the tomographic views of the transverse spatial parton distribution in the nucleon at certain parton longitudinal momentum fractions $x$. Figure adapted from Ref.[8]

This 1+2-dimensional "mixed" longitudinal momentum and transverse position representation provides a set of "tomographic images" of the nucleon as illustrated in the cartoon shown in Fig. 2. More detailed information on GPDs and their properties can be found in Refs. [9-11].

Complementary to the exclusive single photon production, HEMP allows independent access to different bilinear combinations of "meson form factors" linked to the GPDs. They are also convolution of the $x$-dependence of the GPD with the hard scattering kernel and the distribution amplitude to describe the one of the produced meson. Collinear factorisation is rigorously proven for amplitudes with longitudinal polarization of the virtual photon [12]. Vector meson production by longitudinal virtual photons selects only the GPDs $H^{f}$ and $E^{f}$ while scalar meson production selects the GPDs $\tilde{H}^{f}$ and $\tilde{E}^{f}$. In contrast to DVCS both quark and gluon GPDs contribute to the meson production at the same order in $\alpha_{S}$. Decomposition on quark flavor and gluon contributions can be realized through the different combinations obtained with a set of mesons. For example:

$$
\begin{aligned}
& H_{\rho^{0}}=\frac{1}{\sqrt{2}}\left(\frac{2}{3} H^{u}+\frac{1}{3} H^{d}+\frac{3}{8} H^{g}\right) \\
& H_{\omega}=\frac{1}{\sqrt{2}}\left(\frac{2}{3} H^{u}-\frac{1}{3} H^{d}+\frac{1}{8} H^{g}\right) \\
& H_{\phi}=-\frac{1}{3} H^{s}-\frac{1}{8} H^{g}
\end{aligned}
$$

Only Compton or "meson" form factors, which are convolution of GPDs with a the hard scattering kernel, are experimentally accessible. In practice, to extract GPDs embedded in these measured form factors, the strategies are either to constrain models of GPDs against experimental data or to make the most general parametrization the GPDs and to determine the parameters by a global fit to all the available data. The reader can be referred to the original literature for models and constrains by Vanderhaeghen et al. [9, 13], Guidal et al. [14], Diehl et al. [15], Goloskokov and Kroll [16], Kroll, Moutarde and Sabatié [21]. Parametrizations and tentative for global fitting procedure can be found in the references Müller et al. [17-19], Goldstein et al. [20] and Guidal et al. [22]. 
This phenomenology is completed by progress in lattice calculations which predict the moments of the GPDs (see results from the QCDSF-UKQCD [27, 28] and LHPC [29, 30] Collaborations).

\section{Experimental programs}

The goal of the experimental program is to study the GPDs and the nucleon tomography in a large kinematic range from valence quark to sea quarks and gluons. This can only be achieved with several experiments taking advantage of different lepton beam energies.

Already a large number of experiments have been performed for exclusive meson production. They include production of vector mesons as $\rho$ (H1 [36], ZEUS [42], COMPASS [47], HERMES [55], Jlab [64]), $\omega$ (ZEUS [43], JLab [65]), $\phi$ (H1 [37], ZEUS [44], HERMES [56], JLab [66]), $J / \Psi$ (H1 [38], ZEUS [45, 46]), $\Upsilon$ (H1 [39]) and pseudo scalar mesons as $\pi^{0}$ (Jlab [67]) and $\pi^{+}$(HERMES [57], JLab [68]). An overview of DVCS experiments either done in the past at HERA (H1 [31-34], ZEUS [40, 41], HERMES [48-54]) and JLab (6 GeV) (Hall A [58, 59], CLAS [60-63]) or foreseen at present at CERN with COMPASS [69] and JLab (12 GeV) [7075] or projected in a further future with a collider [76-78] is summarized in the Fig. 3 in the kinematic plane $\left(Q^{2}, x\right)$. The main characteristics for beam and target are presented in Table 1 .

Table 1. DVCS experiments

\begin{tabular}{|c|c|c|}
\hline \multicolumn{3}{|c|}{ Collider mode } \\
\hline$<2007$ & $\begin{array}{l}\text { H1, ZEUS } \\
\text { at DESY }\end{array}$ & $\begin{array}{l}\text { polarized } 27.5 \mathrm{GeV} \mathrm{e}^{-} / \mathrm{e}^{+} \\
\& \text { unpolarized } 920 \mathrm{GeV} \text { protons } \\
\quad(\sqrt{s}<318 \mathrm{GeV})\end{array}$ \\
\hline$>2018 ?$ & $\begin{array}{l}\text { EIC } \\
\quad \text { in China }\end{array}$ & $\begin{array}{l}\text { polarized } 3 \mathrm{GeV} \mathrm{e}^{-} \\
\& \text { polarized } 12 \mathrm{GeV} \text { protons } \\
\quad(\sqrt{s}<12 \mathrm{GeV})\end{array}$ \\
\hline$>2025 ?$ & $\begin{array}{l}\text { EIC } \\
\quad \text { in USA }\end{array}$ & $\begin{array}{l}\text { pol. electrons up to } 20 \mathrm{GeV} \\
\& \text { pol. protons up to } 250 \mathrm{GeV} \\
(\sqrt{s}<140 \mathrm{GeV})\end{array}$ \\
\hline \multicolumn{3}{|c|}{ Fixed target experiments } \\
\hline$<2007$ & $\begin{array}{l}\text { HERMES } \\
\text { at DESY }\end{array}$ & $\begin{array}{l}\text { polarized } 27.5 \mathrm{GeV} \mathrm{e}^{-} / \mathrm{e}^{+} \\
\& \text { long. or transv. polarized } \mathrm{p}, \mathrm{d} \\
\quad(\sqrt{s}<7.5 \mathrm{GeV})\end{array}$ \\
\hline $\begin{array}{l}\text { past \& } \\
\text { present }\end{array}$ & $\begin{array}{l}\text { JLab } \\
\quad \text { in USA }\end{array}$ & $\begin{array}{l}\text { polarized } 6 \text { and } 12 \mathrm{GeV} \mathrm{e}^{-} \\
\& \text { unpol., long. or transv. pol. p, } \mathrm{d} \\
\quad(\sqrt{s}<5 \mathrm{GeV})\end{array}$ \\
\hline present & $\begin{array}{l}\text { COMPASS } \\
\text { at CERN }\end{array}$ & $\begin{array}{l}\text { polarized } 160 \mathrm{GeV} \mu^{-} / \mu^{+} \\
\& \text { unpol. p; transv. pol. p? } \\
\quad(\sqrt{s}<18 \mathrm{GeV})\end{array}$ \\
\hline
\end{tabular}

The exclusive single photon production is the golden channel as both the pure DVCS cross section and the DVCS amplitude can be extracted. The hard exclusive single photon production off an unpolarised nucleon tar- get can be written as ${ }^{1}$ :

$$
\begin{aligned}
\frac{\mathrm{d}^{4} \sigma(\ell p \rightarrow \ell p \gamma)}{\mathrm{d} x_{B} \mathrm{~d} Q^{2} \mathrm{~d}|t| \mathrm{d} \phi}= & \mathrm{d} \sigma^{B H}+\left(\mathrm{d} \sigma_{\text {unpol }}^{D V C S}+P_{\ell} \mathrm{d} \sigma_{p o l}^{D V C S}\right) \\
& +e_{\ell}\left(\operatorname{Re} I+P_{\ell} \operatorname{Im} I\right),
\end{aligned}
$$

where $P_{\ell}$ and $e_{\ell}$ are the polarization and the charge of the lepton beam respectively and $I$ is the interference term between DVCS and BH. $\phi$ is the angle between the scattering plane and the photon production plane as presented in Fig.4. The different BH, DVCS and interference contributions are presented in Fig. 5 for the COMPASS muon beam energy of $160 \mathrm{GeV}$ and for three domains in $x_{B}$ for $Q^{2}>1 \mathrm{GeV}^{2}$. Due to the high energy beam, COMPASS as well as the HERA collider offer the advantage to provide various kinematic domains where either $\mathrm{BH}$ or DVCS dominates. In the lower- $x_{B}$ region at COMPASS,

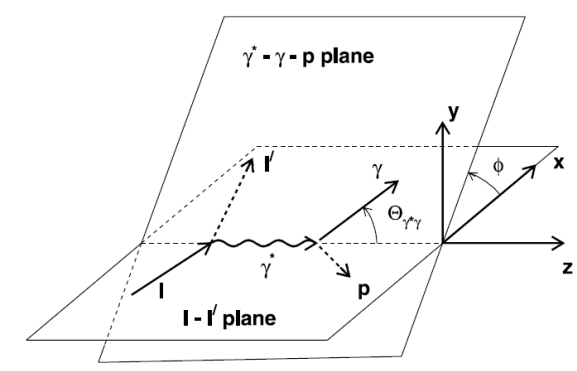

Figure 4. Definition of the azimuthal angle $\phi$ in DVCS in the target rest frame. For $\phi=0$, the real photon is emitted in the same half-plane (with respect to the virtual photon) as the two muons

the almost pure $\mathrm{BH}$ event sample is an excellent reference yield allowing precise monitoring of the global efficiency of the apparatus, as the $\mathrm{BH}$ amplitude is well known (it relies only on the knowledge of elastic nucleon form factors). The practically negligible contribution of the DVCS process in this region is "amplified" by the BH process, leading to a non-negligible contribution of the interference term, which grows with increasing $x_{B}$. Using high lepton energy, in the highest accessible $x_{B}$ region, DVCS events are produced at almost the same rate as $\mathrm{BH}$ events. When, the reasonably small $\mathrm{BH}$ contribution is subtracted, the $\phi$ dependence of the interference is integrated over, measuring the $x_{B}$ dependence of the $t$-slope of the pure DVCS cross section over the full experimentally accessible $x_{B}$ range will allow us to draw conclusions on the "tomographic partonic images" of the nucleon. Using either high or low lepton energy the study of the interference term through measurements of azimuthal dependencies of cross section differences and asymmetries will allow us to constrain various linear combinations of GPDs.

Measurements of hard scattering processes are much more challenging than traditional inclusive or semiinclusive scattering experiments. An ideal experiment is extremely demanding, requiring:

\footnotetext{
${ }^{1}$ For simplicity $\mathrm{d} \sigma$ is used in the following instead of $\frac{\mathrm{d}^{4} \sigma(\mu p \rightarrow \mu p \gamma)}{\mathrm{d} x_{B} \mathrm{~d} Q^{2} \mathrm{~d}|t| \mathrm{d} \phi}$.
} 


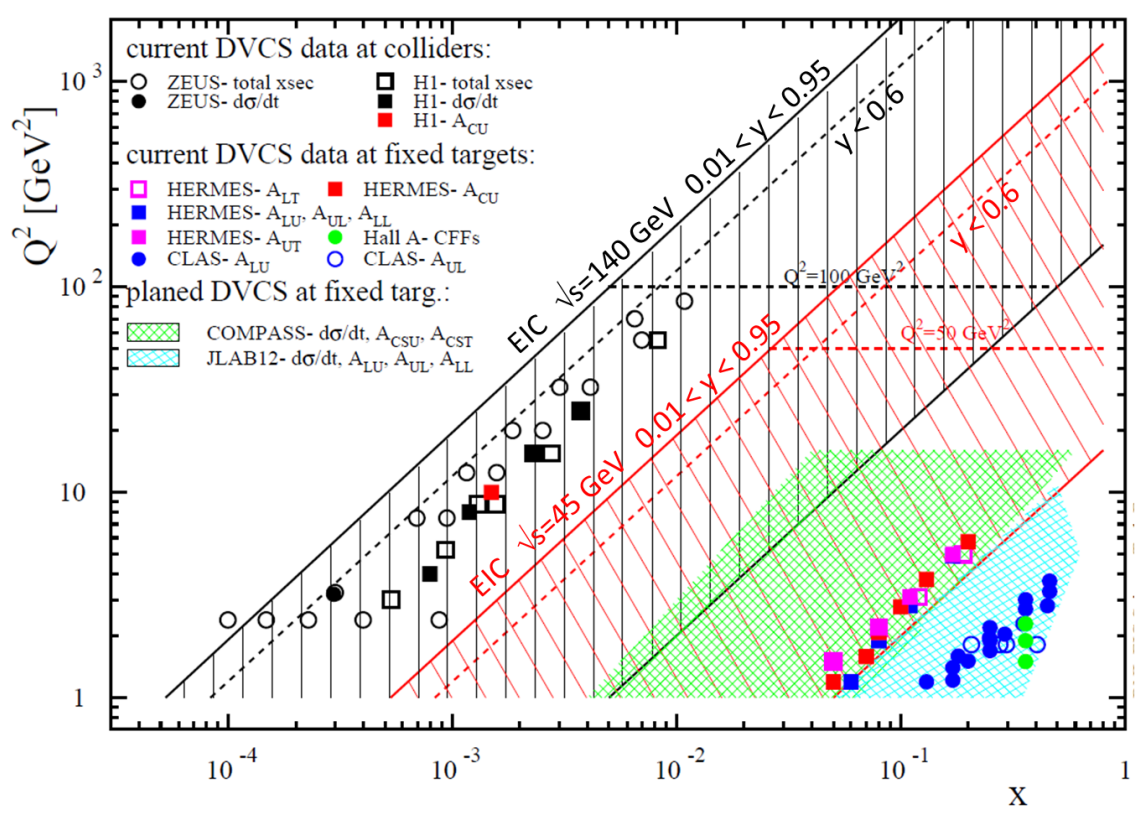

Figure 3. An overview of existing and planned measurements of DVCS in the plane of $Q^{2}$ and the Bjorken variable $x_{B}$. The points are measurements already done at HERA (H1, ZEUS, HERMES) and JLab (6 GeV). The green and blue domains will be investigated at COMPASS and JLab $(12 \mathrm{GeV})$ respectively in the next years. The hatched area using a possible future EIC collider with two energy scenario is also presented. Figure from Ref. [77].
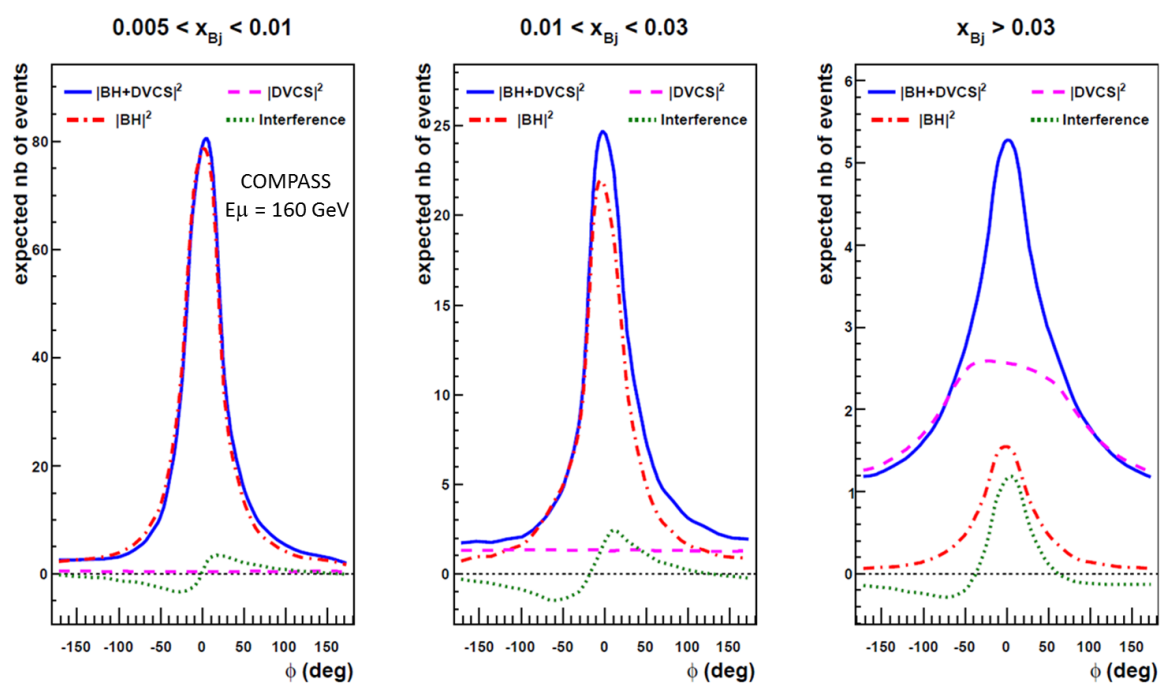

Figure 5. The different BH, DVCS and interference contributions for the exclusive single photon production for three bins in $x_{B}$ for $Q^{2} \leq 1 \mathrm{GeV}^{2}$ using the $160 \mathrm{GeV}$ muon beam available at COMPASS at CERN (Monte Carlo simulation). Figure from Ref. [69].

- high lepton beam energy to insure the hard scattering regime for the applicability of the GPD formalism, to cover a large kinematic domain (over $x_{B}$ and $Q^{2}$ ) and to extract either BH-DVCS interference terms or pure DVCS cross section.

- polarized leptons and availability of positive and negative leptons to play with the different interference terms between the DVCS and BH amplitudes. Using the two states of polarization select the imaginary part of the DVCS amplitude while using the two states of charge select the real part.
- variable lepton energy will allow L/T separation for pseudo scalar meson production and $\epsilon$ separation between interference term and pure DVCS contribution.

- high luminosity for small cross section investigation and fully differential analysis in $\left(x, Q^{2}, t, \phi\right)$. Only Jlab and the future EIC collider in USA have or are foreseen with a comfortable luminosity larger than $10^{33}-10^{34} \mathrm{~cm}^{-2} \mathrm{~s}^{-1}$

- hermetic detector to insure exclusivity of the reaction.

The exclusive processes require a difficult full reconstruction of final state particles. Using a collider, the out- 
going proton is emitted at small $t$ with a high momentum in the very forward direction, so it remains undetected in the beam pipe (except if specific detectors as Roman pots are installed). With a fixed target the proton is emitted at large angle with a small momentum (typically smaller than $1 \mathrm{GeV} / \mathrm{c}$ as dictated by small $t$ ), so it is very challenging to add a detector with sufficiently large acceptance and low momentum detection. So far the experiments have been done mainly without the detection of all three particles. Before installation of a recoil proton detection in fixed target experiments, the missing mass technique is used together with careful background subtraction. The missing mass for DVCS

$$
M_{X}^{2}=\left(k+p-k^{\prime}-q^{\prime}\right)^{2}
$$

where $k, k^{\prime}, p, q^{\prime}$ are the four momenta of the incident and outgoing leptons, target proton at rest, and outgoing real photon respectively, is required to be within an "exclusive region" about the squared proton mass, with boundaries defined by the resolution on $M_{X}^{2}$ mainly given by incident and outgoing leptons and real photon. At HERMES [49-54] with a beam energy of $27 \mathrm{GeV}$ the cut is $-(1.5 \mathrm{GeV})^{2}<\mathrm{M}_{\mathrm{X}}^{2}<(1.7 \mathrm{GeV})^{2}$ as it can be seen in Fig. 6. At JLab Hall A [58] with a beam energy of 5.5 $\mathrm{GeV}$ the cut is $+(0.6 \mathrm{GeV})^{2}<\mathrm{M}_{\mathrm{X}}^{2}<(1.08 \mathrm{GeV})^{2}$. The resolution of the peak is about $0.76 \mathrm{GeV}$ and $0.14 \mathrm{GeV}$ at HERMES and JLab respectively, it varies nearly proportionally to the beam energy. This makes the exclusivity more challenging when the beam energy is high. The following competing reactions with extra photon or pion in addition to $\mathrm{p}\left(e, e^{\prime} \gamma\right) \mathrm{p}$ are:

1. $e p \rightarrow e \pi^{0} p$ for exclusive $\pi^{0}$ production when one photon of $\pi^{0}$ decay is not detected,

2. $e p \rightarrow e \gamma \Delta$ for proton excitation in the resonance $\Delta$ which decays in nucleon and pion not detected,

3. $e p \rightarrow e \gamma N \pi \pi$ (or with more 2 pions production) for semi-inclusive events.

The first contamination is reasonably large at JLab [58, $62,62]$ while it is found negligible at HERMES [49-54]. The determination of exclusive $\pi^{0}$ production where the 2 photon decays are reconstructed in the apparatus allows to estimate the contamination when one photon escapes the detection. The other two contributions are evaluated at HERMES by simulation (as seen in Fig. 6). At JLab Hall A [58] the cut in $M_{X}^{2}$ at a value close to $\left(m_{\text {proton }}+m_{\pi}\right)^{2}$ removes most of this background. A lot of efforts have been made to introduce a recoil detector to check the quality of the exclusive sample. At JLab [58] the exclusive production is found to have less than $3 \%$ contamination. At HERMES [54] the installation of a recoil detector for the data taking in 2006-2007, while reducing the acceptance of the experiment with a proton momentum threshold of $250 \mathrm{MeV} / \mathrm{c}$, allows the elimination of resonant background, that was estimated to contribute an average of about $12 \%$ to the signal in previous HERMES publications [49-53].

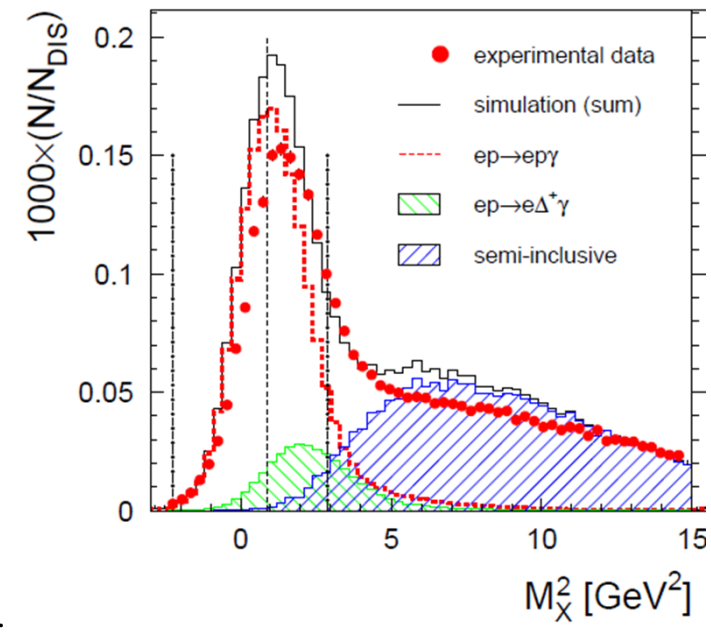

Figure 6. Distributions of the squared missing mass measured at HERMES without recoil detection. The cuts applied to the missing mass to select the most exclusive signal are indicated as vertical dashed-dotted lines. The exclusive signal is expected around the square of the proton mass, indicated by as vertical dashed line. The contribution for $e p \rightarrow e p \gamma$ events is indicated in dashed line, the contributions from resonant production and semi-inclusive background are shown as hatched histograms (simulated distributions). Figure from Ref. [54].

\section{Cross section measurement and evolution as a function of $W$ and $t$}

Exclusive vector meson production has been well studied still a long time. It gives information from the transition from the soft to the hard regime of strong interactions. The soft regime is well described within the Regge phenomenology and Vector Dominance Model. The virtual photon fluctuates in a vector meson which interacts with the nucleon by the exchange of a Pomeron of trajectory:

$$
\alpha_{\mathbb{P}}(t)=\alpha_{\mathbb{P}}(0)+\alpha^{\prime}(t)
$$

A universal parametrization of the soft Pomeron gives $\alpha_{\mathbb{P}}(0)=1.08$ and $\alpha^{\prime}=0.25$. The hard regime is well described by pQCD. The virtual photon splits into a quarkantiquark pair and in the simplest approach of pQCD, two gluons which form a colorless object are emitted from the nucleon and couple to the quark-antiquark pair. The interest in the evolution from soft to hard regime comes from the need to understand at which scale a partonic language is applicable. The exclusive vector meson production can then be related to GPDs and this phenomenology gives a few basis for GPD models.

In the transition from soft to hard regime, the cross section $\sigma$ has the following dependence with the photonproton center of mass energy $W$ and the four-momentum transfer $t$ :

- $d \sigma / d W \propto W^{\delta}$ with $\delta$ varying from a value of about 0.2 in the soft regime to 0.8 in the hard one. The former value is in agreement with the soft Pomeron intercept while the later is in accordance with the steep rise of the gluon density with respect to $W$. 
- $d \sigma / d|t| \propto e^{-B|t|}$ with $B$ varying from a value of about 10 $\mathrm{GeV}^{-2}$ to an asymptotic value of about $5 \mathrm{GeV}^{-2}$. The slope $B$ measures the extension of the transverse size of the scattering objects. The applicability of pQCD implies a small transverse size of the quark-antiquark pair which happens when either $Q^{2}$ or the mass of the quark gets large and so the slope $B$ is determined only by the proton size. The dominance of small-size configurations is illustrated by the convergence of the different slopes at large $Q^{2}$ or large meson mass. The hard regime is reached at high $Q^{2}$ for light vector mesons as $\rho, \phi$ and directly from photoproduction for heavier mesons as $J / \psi, \Upsilon$ (see Fig. 7).

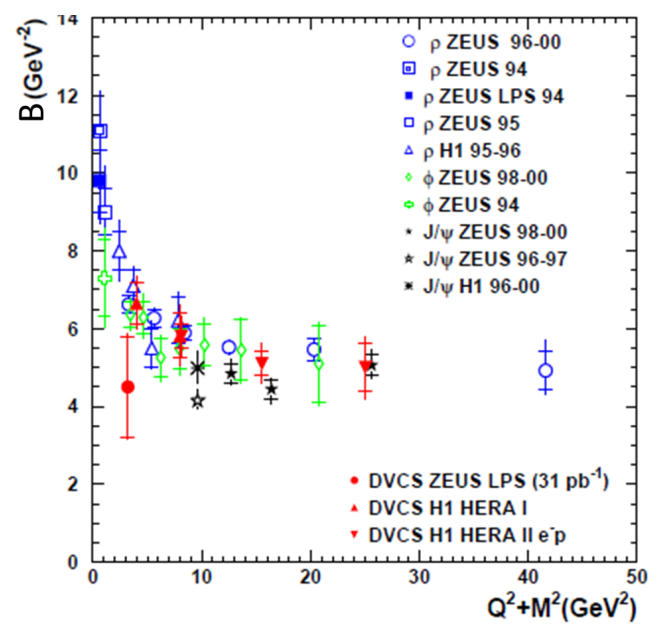

Figure 7. A compilation of $B$ slope values for selected vector meson as a function of $Q^{2}+M^{2}$ where $M$ is the mass of the vector meson. Also DVCS results are included.

In hard regime where Regge phenomenology is not applicable an effective Pomeron trajectory may nevertheless be extracted. In the Regge formalism the differential cross section can be expressed as:

$$
d \sigma / d t \propto e^{-B_{0}|t|} \times\left(\frac{W}{W_{0}}\right)^{4\left(\alpha_{\mathbb{P}}(t)-1\right)}
$$

so the exponential $t$-slope B of $d \sigma / d t$ depends on $W$ as:

$$
B(W)=B_{0}+4 \alpha^{\prime} \ln \left(W / W_{0}\right)
$$

In the low regime there is clearly an interplay between the $t$ and $W$ dependence of the differential cross section due to the non-zero value of $\alpha^{\prime}$. When $W$ increases, $B$ increases which means that the scattering object get bigger. This fact is called shrinkage. In the hard regime the value of $\alpha^{\prime}$ is found rather small which means that there is no or little shrinkage. For illustration Fig. 8 shows the effective Pomeron trajectories extracted for $J / \psi$ production for photoproduction and for $<Q^{2}>=6.8 \mathrm{GeV}^{2}$ by the ZEUS experiment [46].

Thanks to the high beam energy available at the HERA collider the experiments H1 [31-34] and ZEUS [40, 41] have measured the pure DVCS cross section in a large domain of $x_{B}$ ranging from $10^{-4}$ to $10^{-2}$ (see Fig.3). In this

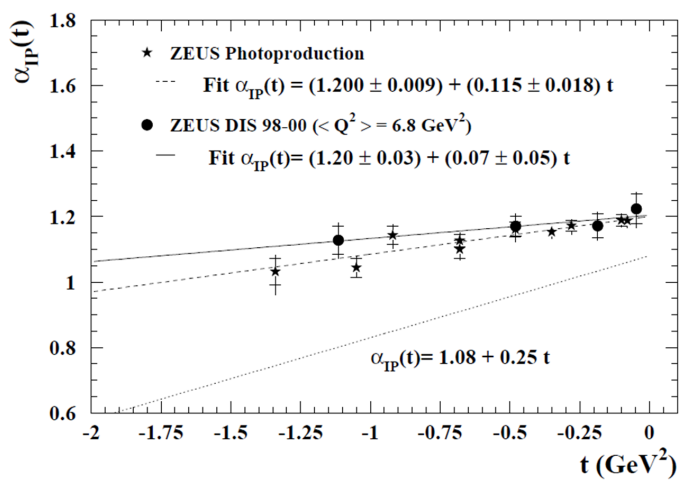

Figure 8. Effective Pomeron trajectories extracted for $J / \psi$ production for $<Q^{2}>=0 \mathrm{GeV}^{2}$ (dashed line) and for $<Q^{2}>=6.8$ $\mathrm{GeV}^{2}$ (solid line) by the ZEUS experiment. The dotted line for the soft Pomeron trajectory is indicated for comparison. Figure from Ref.[46].

small $x_{B}$ domain two-gluon exchange plays a major role in addition to the leading order quark-photon scattering process. Fig. 9 (left panel) shows the differential cross section $d \sigma / d t$ for different ranges in $Q^{2}$ measured (integrated over $W)$ at H1 [33] and ZEUS [41]. The data are well described by the exponential behavior and the parameter $B$ of the $t$ slope is presented on the right panel. The $Q^{2}$ dependence can be parametrized as $B\left(Q^{2}\right)=A\left(1-B^{\prime} \log \left(\mathrm{Q}^{2} / 2 \mathrm{GeV}^{2}\right)\right)$ with $A=6.98 \pm 0.54 \mathrm{GeV}^{2}$ and $B^{\prime}=0.12 \pm 0.03 \mathrm{GeV}^{2}$. The average slope is $B=5.45 \pm 0.19_{\text {stat }} \pm 0.34_{\text {syst }} \mathrm{GeV}^{-2}$ at $<Q^{2}>=8 \mathrm{GeV}^{2}$ which corresponds to a transverse extension of sea quarks and gluons in the proton of $\sqrt{<r_{T}^{2}>}=$ $0.65 \pm 0.02 \mathrm{fm}$ for an average value $x_{B}=1.210^{-3}$. This can be compared to the transverse charge radius of the proton $\sqrt{\left.<r_{T}^{2}\right\rangle_{c h}}$ given either by $\sqrt{\left.4 \frac{d}{d t} G_{E}^{p}\right|_{t=0}}=0.72 \pm 0.01 \mathrm{fm}$ or by $\sqrt{\left.4 \frac{d}{d t} F_{1}^{p}\right|_{t=0}}=0.67 \pm 0.01 \mathrm{fm}$. Moreover the value of $B$ as a function of $W$ show no significant dependence (left panel on bottom).

Thanks to the high energy available at COMPASS, the DVCS cross section can also be extracted after BH subtraction and integration of the azimuthal dependence. Fig. 10 (on top) shows the projected statistical accuracy for a measurement at COMPASS of the $x_{B}$-dependence of the $t$-slope parameter $B\left(x_{B}\right)$ of the DVCS cross section. In the simple ansatz $\frac{d \sigma}{d t} \propto \exp \left(-B\left(x_{B}\right)|t|\right)$ with $B\left(x_{B}\right)=B_{0}+2 \alpha^{\prime} \log \left(\frac{x_{0}}{x}\right)$. At small $x_{B}$, no evolution with $x_{B}$ was observed at HERA [33]. In the valence region, where no experimental determinations of $B$ exist, some information comes from fits adjusted to form factor data which give $\alpha^{\prime} \simeq 1 \mathrm{GeV}^{2}[14,15]$. For the simulation two values $\alpha^{\prime}=0.125$ and $\alpha^{\prime}=0.26$ are shown which correspond to the half and the total of the value for Pomeron exchange in soft scattering processes. These values can be determined at COMPASS with an accuracy better than 2.5 sigma. This will provide information on the evolution of transverse size of the nucleon $\left(\left\langle r_{\perp}^{2}\left(x_{B}\right)\right\rangle \approx 2 \cdot B\left(x_{B}\right)\right.$ at small $x_{B}$ ) over the uncharted $x_{B}$-range from 0.01 to 0.1 

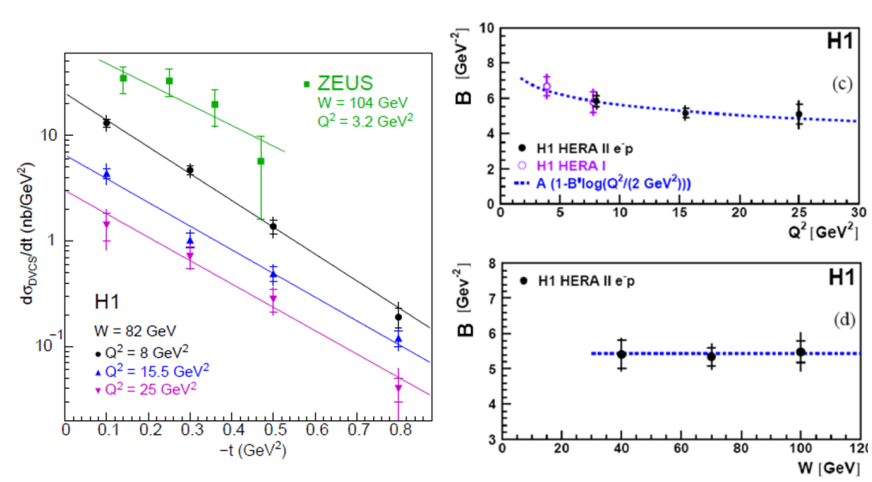

Figure 9. Left: The $t$-dependence of the DVCS cross section for several values of $Q^{2}$ as measured by $\mathrm{H} 1$ and ZEUS. The curves are results of fits of the form $e^{-B|t|}$. Right: the fitted B values as a function of $Q^{2}$ (top) and $W$ (bottom). The curves represent the result of the fit $B\left(Q^{2}\right)=A\left(1-B^{\prime} \log \left(\mathrm{Q}^{2} / 2 \mathrm{GeV}^{2}\right)\right)$ (top) and of the average value $B=5.45 \mathrm{GeV}^{-2}$ (bottom). Figure from Ref. [33].
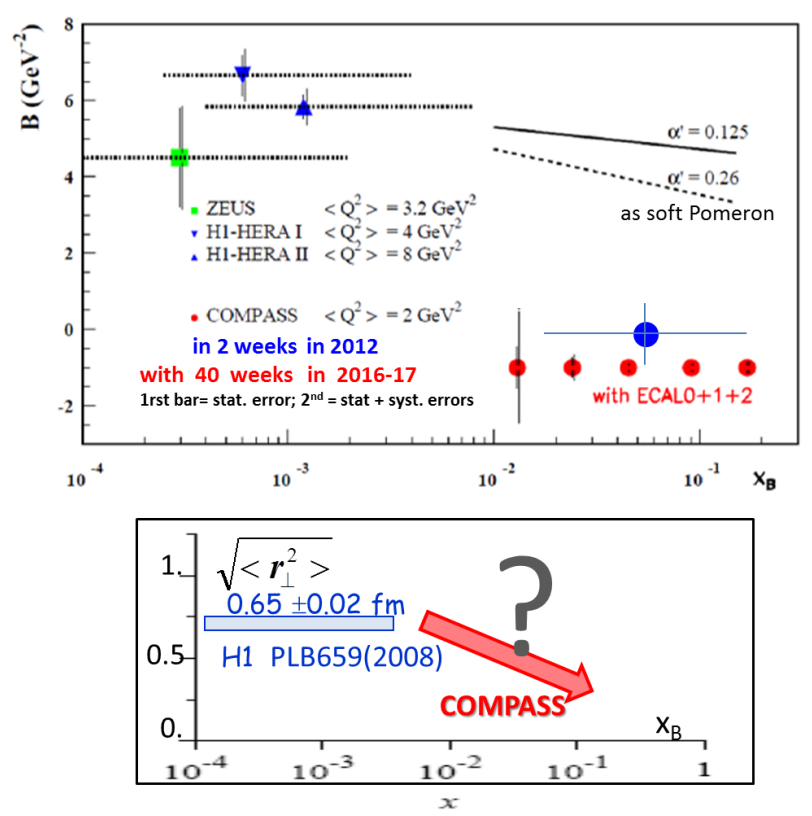

Figure 10. Top: Projections in 280 days for measuring the $x_{B}$ dependence of the $t$-slope parameter $B\left(x_{B}\right)$ of the DVCS cross section, calculated for $1<Q^{2}<8 \mathrm{GeV}^{2}$. A comparison to HERA results with similar $\left\langle Q^{2}\right\rangle$ is shown. The left vertical bar on each data point indicates the statistical error only while the right one includes also the systematic uncertainty. The blue point indicates the statistical error which can be reached using the 4 weeks pilot run done in 2012. Two different parameterisations are shown using $\alpha^{\prime}=0.125 \mathrm{GeV}^{-2}$ and $0.26 \mathrm{GeV}^{-2}$. Bottom: Transverse proton radius as a function of $x_{B}$ from HERA and COMPASS.

(see Fig. 10 on bottom) further elucidating the issue of "nucleon tomography".

Kumericki and Mueller [18] proposed a flexible parametrization of the GPDs (noted KM09). It is based on both a Mellin-Barnes integral and dispersion integral representation with weakly entangled skewness and $t$ depen- dences. Fig. 11 presents the rather good quality of a simultaneous fit to the DVCS cross sections as a function of $Q^{2}$, $W$ and $t$ (39 data points) measured at HERA [32, 33, 40] and DIS [79] (85 data points) data which is performed in the CS scheme to NNLO accuracy.
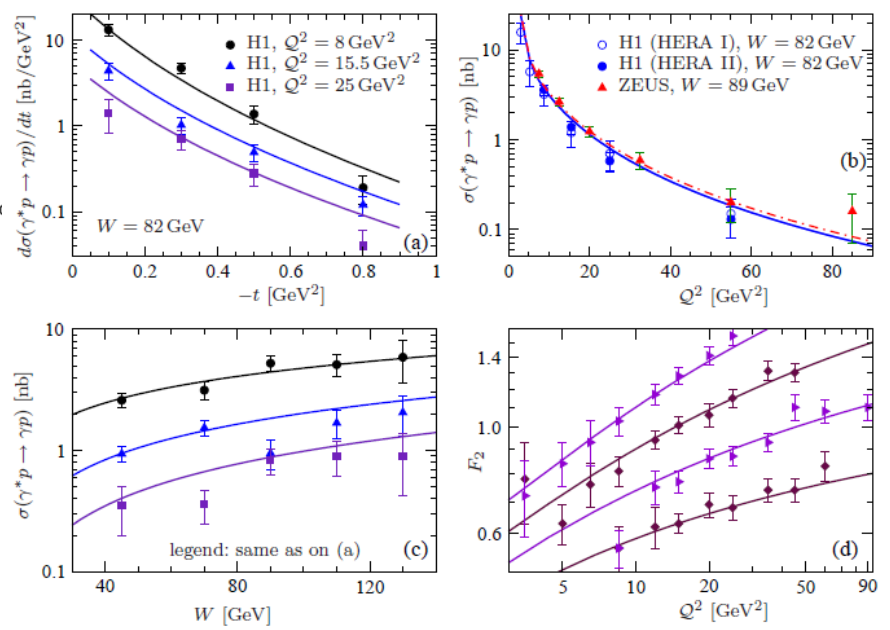

Figure 11. Simultaneous fit to the DVCS and DIS data in the CS scheme at NNLO. First three panels: DVCS cross section measured at $\mathrm{H} 1$ [32, 33] and ZEUS [40] as a function of $t, Q^{2}$ and $W$. Last panel: $F_{2}\left(x_{B}, Q^{2}\right)$ versus $Q^{2}$ for $x_{B}=8.10^{-3}, 3.2$ $10^{-3}, 1.3 .10^{-3}, 5.10^{-4}$ [79]. Figure from Ref. [18].

Goloskokov and Kroll [16] have developed a model of GPDs (noted the GK model) whose parameters are constrained by hard exclusive meson production. Fig. 12 shows the ratio of the longitudinal cross section $\phi$ and $\rho$ production for data measured at high $W$ at $\mathrm{H} 1[36,37]$ and ZEUS [42, 44] and smaller $W$ at HERMES [55, 56]. At HERA at large $W$ and large $Q^{2}$ the data are not far from the symmetry limit $2 / 9$ which can be clearly observed in Eqs. (6) when the gluon contribution dominates. The $Q^{2}$ dependence at HERA is completely determined by the flavor symmetry breaking factor $\kappa_{S}$ between the sea quarks $\bar{u}, \bar{d}$ and $\bar{s}$ quarks $\left(\bar{u}(x)=\bar{d}(x)=\kappa_{S} \bar{s}(x)\right)$. At smaller $W$ at HERMES the valence quarks contributions gives additional suppression of the ratio.

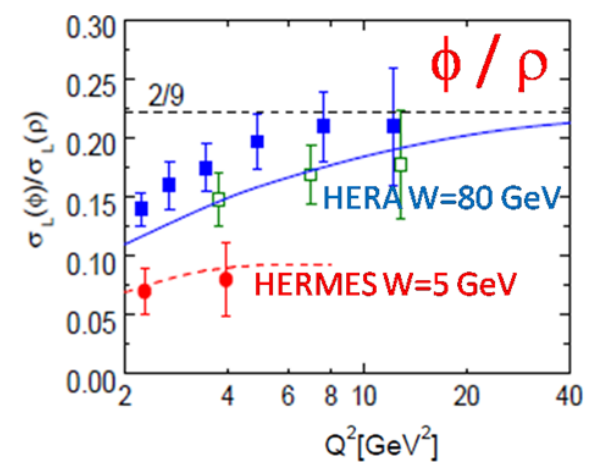

Figure 12. The ratio of the longitudinal cross section $\phi$ and $\rho$ production. Data from H1 [36, 37], ZEUS [42, 44] and HERMES [55, 56]. The solid (dashed) line represents the handbag predictions at $W=75(5) \mathrm{GeV}$. Figure from Ref. [16] (2007). 


\section{Study of the GPD H through interference of DVCS on the proton with Bethe-Heitler}

The real and imaginary parts can be accessed separately through the $\phi$ azimuthal dependence of the interference between DVCS and Bethe-Heitler processes. This method has been proposed by Diehl et al. [80, 81] and well established up to twist-3 contributions by Belitsky, Mueller and Kirchner [82]. Let us consider an unpolarized target and a lepton beam of charge $e_{\ell}$ and longitudinal polarization $P_{\ell}$ to see how the harmonic analysis in $\phi$ can filter the DVCS amplitudes. The harmonic expansion of all the terms of Eq. (7) is:

$$
\begin{aligned}
\mathrm{d} \sigma^{B H} & =\frac{\Gamma\left(x_{B}, Q^{2}, t\right)}{P_{1}(\phi) P_{2}(\phi)}\left(c_{0}^{B H}+c_{1}^{B H} \cos \phi+c_{2}^{B H} \cos 2 \phi\right) \\
\mathrm{d} \sigma_{\text {unpol }}^{D V C S} & =\frac{e^{6}}{y^{2} Q^{2}}\left(c_{0}^{D V C S}+c_{1}^{D V C S} \cos \phi+c_{2}^{D V C S} \cos 2 \phi\right) \\
\mathrm{d} \sigma_{\text {pol }}^{D V C S} & =\frac{e^{6}}{y^{2} Q^{2}}\left(s_{1}^{D V C S} \sin \phi\right) \\
\operatorname{Re} I & =\frac{e^{6}}{x y^{3} t P_{1}(\phi) P_{2}(\phi)}\left(c_{0}^{I}+c_{1}^{I} \cos \phi\right. \\
\operatorname{Im} I & \left.=\frac{\left.e_{2}(\cos 2 \phi)+c_{3}^{I} \cos 3 \phi\right)}{x y^{3} t P_{1}(\phi) P_{2}(\phi)}\left(s_{1}^{I} \sin \phi+s_{2}^{I} \sin 2 \phi\right)\right)
\end{aligned}
$$

where $P_{1}(\phi)$ and $P_{2}(\phi)$ represent the well known $\phi$ dependence of the $\mathrm{BH}$ lepton propagators and

- $c_{0}^{B H}, c_{1}^{B H}, c_{2}^{B H}$ are calculable in QED as far as the proton form factors are known,

- $c_{0}^{D V C S}, c_{0}^{I}, c_{1}^{I}, s_{1}^{I}$ are related to certain combinations of Compton Form Factors representing the leading twist-2 contributions of the helicity Compton amplitude $M^{11}$ for DVCS (with helicity conservation between initial and final state photons),

- $c_{1}^{D V C S}, s_{1}^{D V C S}, c_{2}^{I}, s_{2}^{I}$ are twist-3 contributions of the longitudinal-transverse amplitude $M^{01}$ (with singlehelicity flip),

- $c_{2}^{D V C S}, c_{3}^{I}$ are twist-2 contributions of the transversetransverse amplitude $M^{-11}$ (with double helicity flip possible for the gluons GPDs).

Using electron beams of either opposite polarization or opposite charge, the difference of the cross sections provides either the imaginary part or the real part of the interference term $I$.

$$
\begin{aligned}
\mathrm{d} \sigma^{\leftarrow}-\mathrm{d} \sigma^{\rightarrow} & =2\left[\mathrm{~d} \sigma_{\text {pol }}^{D V C S}+\operatorname{Im} I\right] \stackrel{\text { L.T. }}{\longrightarrow} s_{1}^{I} \sin \phi \\
\mathrm{d} \sigma^{+}-\mathrm{d} \sigma^{+} & =2[\operatorname{Re} I] \stackrel{L . T}{\longrightarrow} c_{0}^{I}+c_{1}^{I} \cos \phi
\end{aligned}
$$

Note that the sum of the cross sections with opposite polarization give also a sensitivity to the real part of the interference term $I$ (as for Fig. 13)

$$
\begin{aligned}
\mathrm{d} \sigma^{\leftarrow}+\mathrm{d} \sigma^{\rightarrow} & =2\left[\mathrm{~d} \sigma^{B H}+\mathrm{d} \sigma_{\text {unpol }}^{D V C S}+\operatorname{Re} I\right] \\
& \stackrel{\text { L.T. }}{\longrightarrow} 2 \mathrm{~d} \sigma^{B H}+c_{0}^{D V C S}+c_{0}^{I}+c_{1}^{I} \cos \phi
\end{aligned}
$$

Using positive and negative muon beams with naturally opposite polarization the sum or the difference of the cross sections provide the imaginary part or the real part of the interference term $I$.

$$
\begin{aligned}
& \mathrm{d} \sigma^{\stackrel{+}{\leftarrow}}-\mathrm{d} \sigma^{-}=2\left[\mathrm{~d} \sigma_{\text {pol }}^{D V C S}+\operatorname{Re} I\right] \\
& \stackrel{\text { L.T. }}{\longrightarrow} c_{0}^{I}+c_{1}^{I} \cos \phi \\
& \mathrm{d} \sigma^{\stackrel{+}{\leftarrow}}+\mathrm{d} \sigma^{\leftrightarrows}=2\left[\mathrm{~d} \sigma^{B H}+\mathrm{d} \sigma_{\text {unpol }}^{D V C S}+\operatorname{Im} I\right] \\
& \stackrel{\text { L.T. }}{\longrightarrow} 2 \mathrm{~d} \sigma^{B H}+c_{0}^{D V C S}+s_{1}^{I} \sin \phi
\end{aligned}
$$

$c_{1}^{I}$ and $s_{1}^{I}$ are the real part and the imaginary part respectively of a combination of Compton form factors:

$$
\left(F_{1} \mathcal{H}+\xi\left(F_{1}+F_{2}\right) \tilde{\mathcal{H}}-t / 4 m^{2} F_{2} \mathcal{E}\right)
$$

where $F_{1}$ and $F_{2}$ are the Dirac and Pauli form factors. The small values of the kinematic factors $\xi$ and $t$ give a dominant contribution of the GPD $H$ when using a proton target. The contribution of the GPD $E$ remains of the order of a few $\%$ while the contribution of the GPD $\tilde{H}$ increases with $x_{B}$ ranging 0.12 at HERMES, 0.21 at Jlab CLAS and even 0.47 at Jlab Hall A[21]. To go further, using longitudinally polarized target give asymmetry providing the combination of Compton form factors:

$$
F_{1} \tilde{\mathcal{H}}+\xi\left(F_{1}+F_{2}\right) \mathcal{H}+\ldots
$$

in order to measure contribution of the GPD $\tilde{H}$. Using a transversely polarized target give asymmetry providing the combination of Compton form factors:

$$
F_{2} \mathcal{H}-F_{1} \mathcal{E}+\ldots
$$

in order to reveal the contribution of the GPD $E$. It may also be accessed through the beam polarization asymmetry using a neutron (or deuterium) target as it can be seen in the previous equation (18) because the neutron form factor $F_{1}$ is negligible compared to $F_{2}$.

The two first published GPD-related experimental results were beam spin asymmetries in DVCS measured using 4.3 GeV polarized electrons at JLab with CLAS [60] and using $27.5 \mathrm{GeV}$ polarized positrons at HERMES [48]. In both experiments significant sinusoidal modulations have been observed showing the dominance of the leading twist handbag diagram.

Fig. 13 shows the absolute difference and sum of cross sections using opposite polarization of beams at Jlab in Hall A [58] at different $t$ bins for $x_{B}=0.36$ and $Q^{2}=$ $2.3 \mathrm{GeV}^{2}$. The two measurements are related to Eq. (13) and Eq. (15). The difference is mainly driven by a sinusoidal modulation with the CFF $\operatorname{Im} \mathcal{H}$. The sum contains contributions of $\mathrm{BH}$, pure DVCS and interference term related to the CFF $\operatorname{Re} \mathcal{H}$. This very challenging measurements of absolute cross sections is realized thanks to a good knowledge of the magnetic spectrometers in Hall A, of the calorimeter and of the luminosity. The difference of cross sections is rather well reproduced by the recent predictions of Kroll, Moutarde and Sabatié (KMS) [21]. They use the GK model of GPDs whose parameters have 

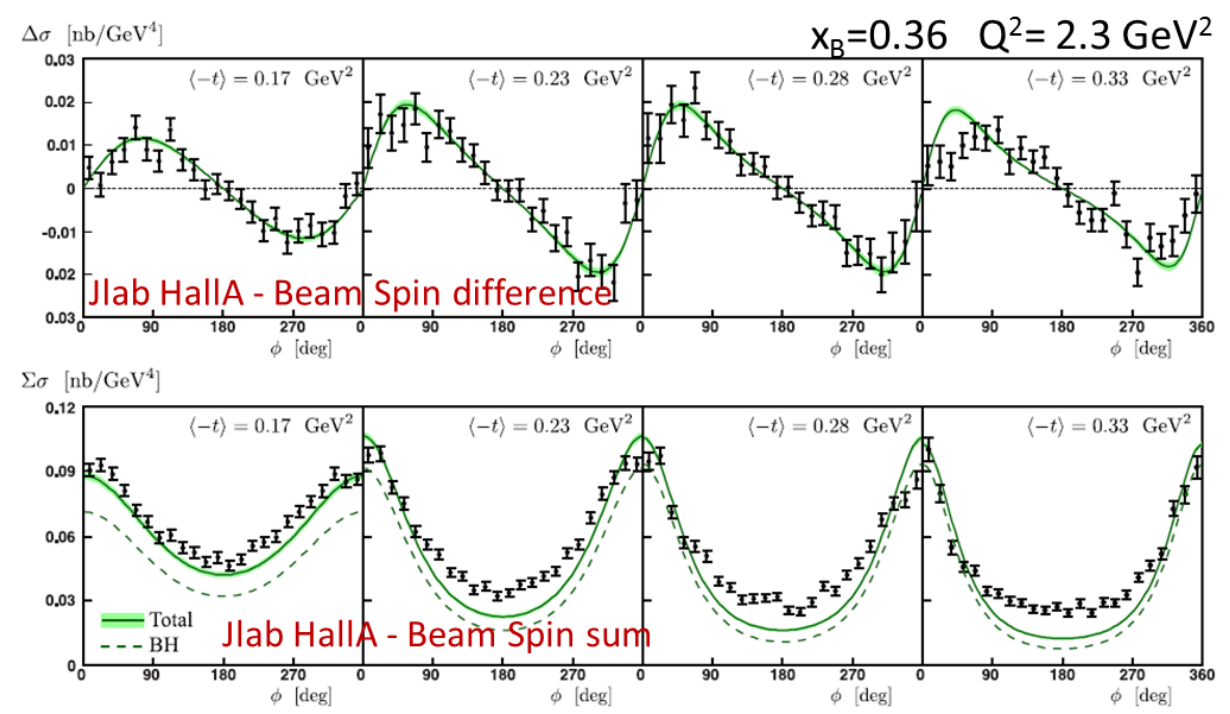

Figure 13. Helicity dependent cross sections measured at Jlab in Hall A [58] at different $t$ bins for $x_{B}=0.36$ and $Q^{2}=2.3 \mathrm{GeV}^{2}$. Top: difference of cross sections for opposite electron helicities versus $\phi$. Bottom: unpolarized cross sections or half the sum of cross sections for opposite electron helicities. BH contribution (dotted line), prediction with the GK model done by Kroll, Moutarde and Sabatié [21] (solid line). Figure from Ref. [21].

been constrained only by Hard Exclusive Meson production. The goal is to apply this model of GPDs to the DVCS case measured at JLab, HERMES, COMPASS and HERA to check the universality of the GPDs. However there is a discrepancy between theory and experiment for the sum of cross sections of up to $30 \%$. Such a tension exists also for calculations from a global fit with the flexible parametrization KM09 of Kumericki and Mueller [18]. Corrections including kinematic effects due to finite $t$ and target mass have been performed without success [23]. A new reanalysis of the data is also being done.

Beam Spin Asymmetries using opposite electron helicities have been measured at JLab with CLAS [62, 63]. An intensive work for a determination of the absolute difference and sum of the cross sections to disentangle all the different contributions is also in progress. However the task is complicated due to the non trivial acceptance of CLAS.

Beam Spin and Beam Charge Asymmetries have been measured at HERMES using polarized electrons and positrons of $27 \mathrm{GeV}$ on an unpolarized proton target [53] without recoil proton detection. The dominant modulations (see Eqs. (13) and (14)) $s_{1}^{I}$ noted $A_{L U, I}^{\sin \phi}, c_{0}^{I}$ noted $A_{C, I}^{\cos (0 \phi)}, c_{1}^{I}$ noted $A_{C, I}^{\cos \phi}$, are presented as a function of $|t|$, $x_{B}$ and $Q^{2}$ in Fig. 14 and are compared to predictions from two parametrizations of GPDs. The solid and dashed curves show calculations (KM09a and KM09b) by Kumericki and Mueller [18] from a global fit of the flexible GPD parametrization to all experimental DVCS data from HERA, HERMES and JLab. The results from JLab HAll A are not included in the solid line (KM09a). The dasheddotted curves show calculations (GGL11) by Goldstein, Hernandez and Luiti. [20]. This parametrization fitted also to all the data is based on a quark-diquark model with a Regge-inspired term to determine the $t$-dependence. Fig. 15 shows the same experimental results as a func-
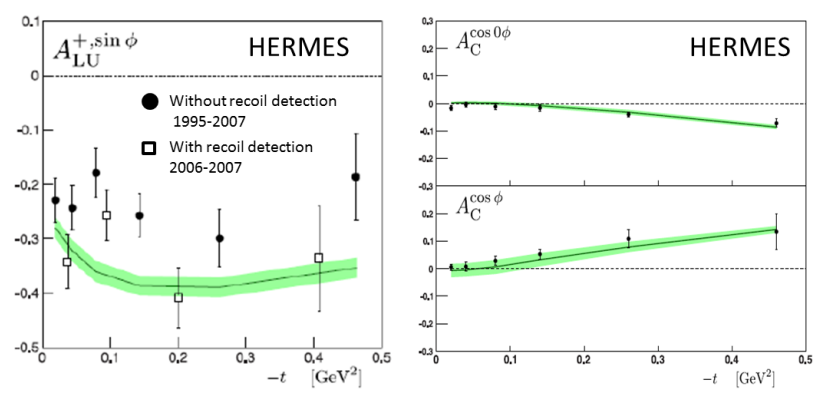

Figure 15. The dominant modulations $s_{1}^{I}$ noted $A_{L U, I}^{\sin \phi}, c_{0}^{I}$ noted $A_{C, I}^{\cos (0 \phi)}, c_{1}^{I}$ noted $A_{C, I}^{\cos \phi}$ for Beam Spin and Beam Charge Asymmetries [53] measured at HERMES using polarized electrons and positrons of $27 \mathrm{GeV}$ on an unpolarized proton target. The open points on left panel correspond to the use of the recoil proton detection [54]. Comparison to predictions by the GK model done by Kroll, Moutarde and Sabatié [21]. Figure from Ref. [21].

tion of $|t|$ and the comparison to predictions using the GK model by Kroll, Moutarde and Sabatié [21]. The new experimental results [54] with the use of the recoil proton detector are also shown on left. They quantify the impact of the absence of recoil detection for all the previous published results. A clear dilution of the measured effect due to background contamination was observed. The leading asymmetry amplitude is varying from $-0.25 \pm 0.019_{\text {stat }} \pm$ $0.047_{\text {syst }}$ to $-0.328 \pm 0.027_{\text {stat }} \pm 0.045_{\text {syst }}$ when adding the recoil proton detection [54].

Fig. 16 shows the results for the imaginary part of the CFF $\mathcal{H}$ which has been tentatively extracted as a func- 


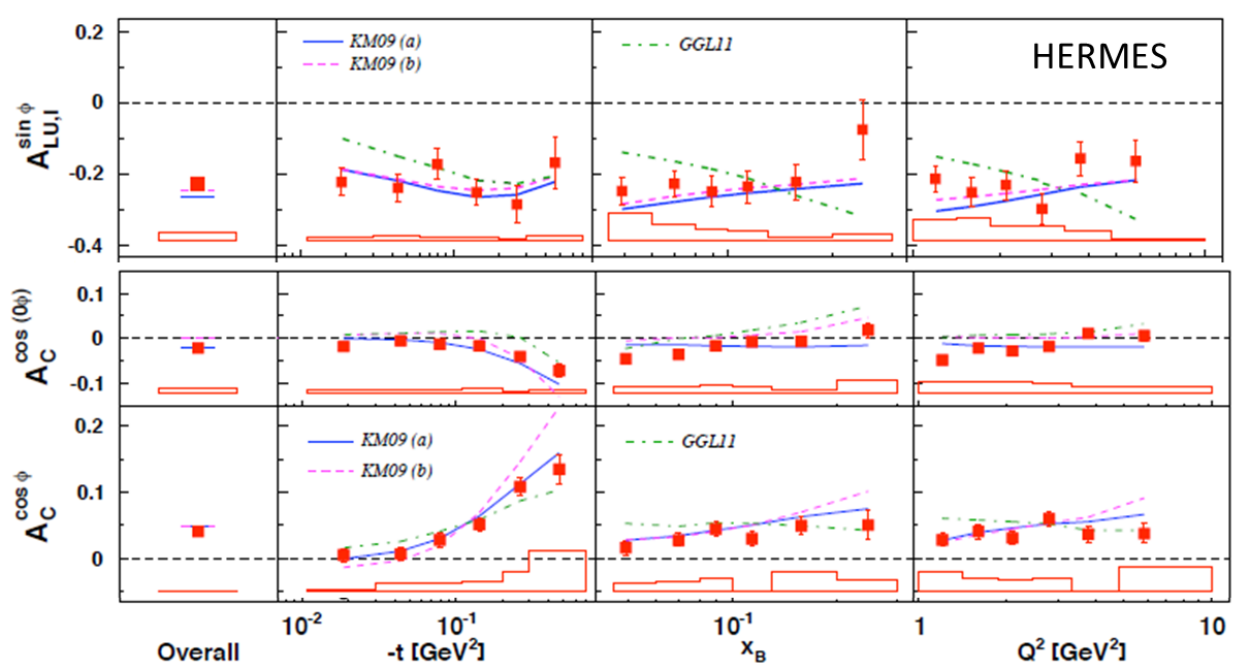

Figure 14. The dominant modulations $s_{1}^{I}$ noted $A_{L U, I}^{\sin \phi}, c_{0}^{I}$ noted $A_{C, I}^{\cos (0 \phi)}, c_{1}^{I}$ noted $A_{C, I}^{\cos \phi}$ for Beam Spin and Beam Charge Asymmetries [53] measured at HERMES using polarized electrons and positrons of $27 \mathrm{GeV}$ on an unpolarized proton target. Comparison to calculations from fits of GPDs by Kumericki and Mueller (KM09a and KM09b) [18] and Goldstein, Hernandez and Luiti (GGL11) [20]. Figure from Ref. [53].

tion of $|t|$ from different local fits [22, 24-26] applied for each of the 3 experiments done at JLab Hall A, CLAS and HERMES. The extracted CFFs $\operatorname{Im} \mathcal{H}$ are compared to predictions (solid red lines) done with the VGG model. The VGG model has been developed by Vanderhaeghen, Guichon and Guidal [13] in 1998 and was evolving [9, 14]. The extracted CFF are also compared to latest calculations by Kumericki and Mueller [18] from a global fit of the flexible GPD parametrization to all experimental DVCS data from HERA, HERMES and JLab. The bottom panel shows a flavor of the "tomographic images" which can be extracted from the $t$-evolution of the imaginary part of the CFF $\mathcal{H}$.
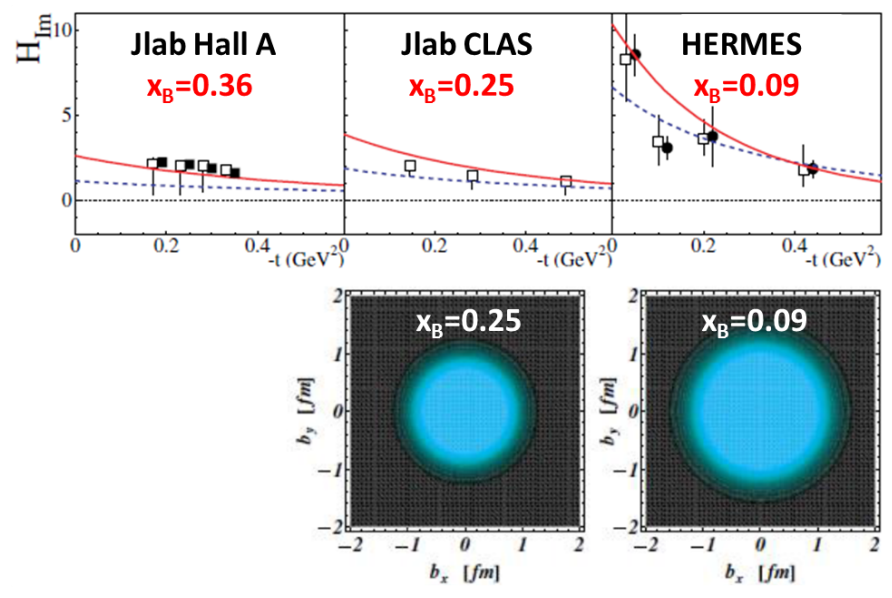

Figure 16. Top: Imaginary part of the CFF $\mathcal{H}$ extracted from different local fits applied for each of the 3 experiments done at JLab Hall A, CLAS and HERMES. Comparison to calculations from a global fit using all the DVCS data (dashed blue line) and to predictions by the VGG model (red solid line). Bottom: "tomographic images" which can be extracted from the $t$-evolution of the imaginary part of the CFF $\mathcal{H}$. Figures are from Ref. [22].
With the $12 \mathrm{GeV}$ beam which will be soon available at Jlab, the goal will be to perform very accurate measurements (in absolute value) using the magnetic spectrometers of Hall A [70] and C [71] associated to highperformance calorimeters and to span with asymmetry measurements the kinematic domain $0.2<x_{B}<0.6$ with $Q^{2}$ varying from 1 (3) to 5 (8) $\mathrm{GeV}^{2}$ using CLAS12 (as can be seen in Fig. 17). The variations of the $t$-dependences across the $x_{B}$ range encode information on "nucleon imaging" of quark position in the transverse plane, which will be extracted via local and global analyses.

The difference and sum of cross sections using the polarized positive and negative $160 \mathrm{GeV}$ muon beams available at COMPASS at CERN will be measured at COMPASS during 2 times 6 months of data taking foreseen in 2016 and 2017. The almost complete setup comprising the COMPASS spectrometer equipped with three calorimeters (one is newly built) and a recoil proton detector CAMERA surrounding a $2.5 \mathrm{~m}$ long hydrogen target has already been used successfully during a pilot run of one month in 2012 . The cumulated statistics in 2016 and 2017 will permit to study 6 bins in $x_{B}$ ranging from 0.005 to $0.3,6$ bins in $t$ ranging from 0.06 to $0.7 \mathrm{GeV}^{2}$ and 4 bins in $Q^{2}$ from 1 to $16 \mathrm{GeV}^{2}$.

Fig. 19 shows the projected statistical accuracy for the measurement in one of the bins. It corresponds to the kinematics $x_{B} \sim 0.05, Q^{2} \sim 2 \mathrm{GeV}^{2}$ for the complete range in $t$ from 0.06 to $0.64 \mathrm{GeV}^{2}$. The back and cyan curves are predictions using the VGG model $[9,13,14]$ and 2 shrinkage parameters $\alpha^{\prime}$ of 0.8 or 0.05 . The large value is well adapted for the valence region. The red and blue curves (shown both on left and in the zoom on right) are calculations from a global fit to present world data [18] with (blue) and without (red) including JLab Hall A data. The green curve (shown in the zoom on right) has been recently evaluated using the GK model [21]. Fig. 20 presented the 


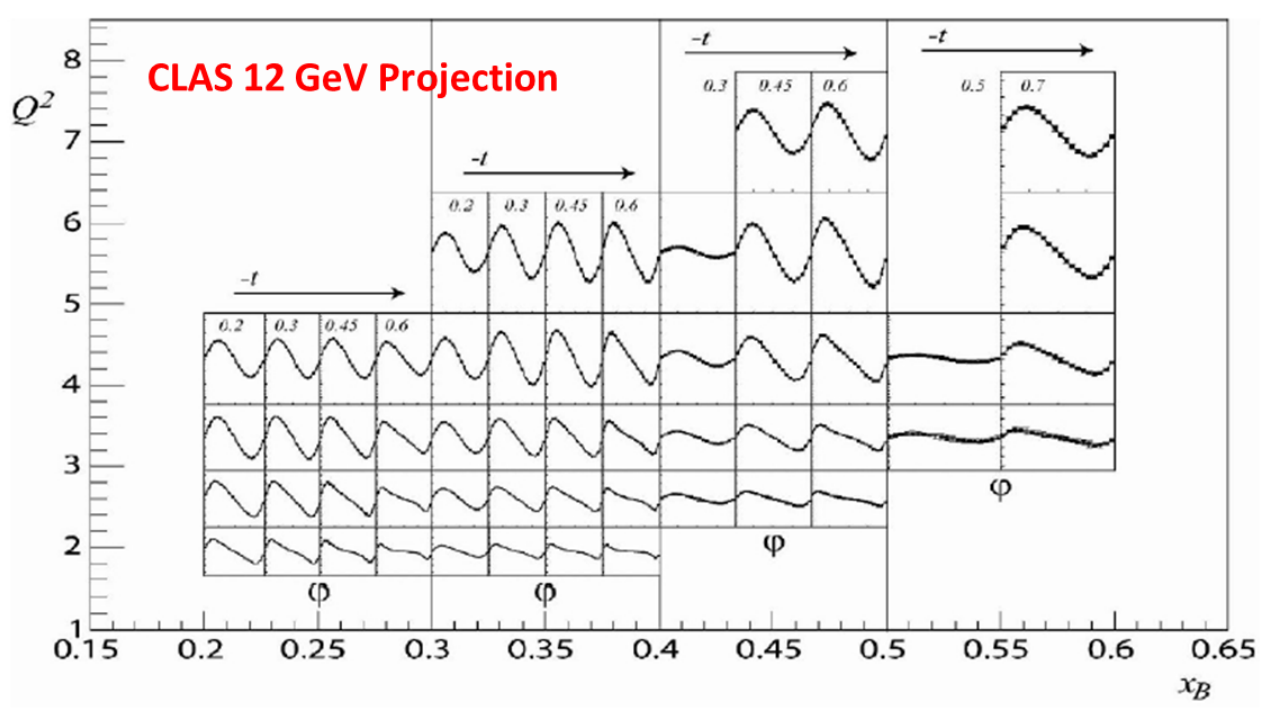

Figure 17. The projected asymmetry measurements for 80 days of running time in the domain $0.2<x_{B}<0.6$ and $Q^{2}$ varying from 1 (3) to 5 (8) $\mathrm{GeV}^{2}$ using JLab $12 \mathrm{GeV}$ and CLAS12. Figure from Ref. [72].

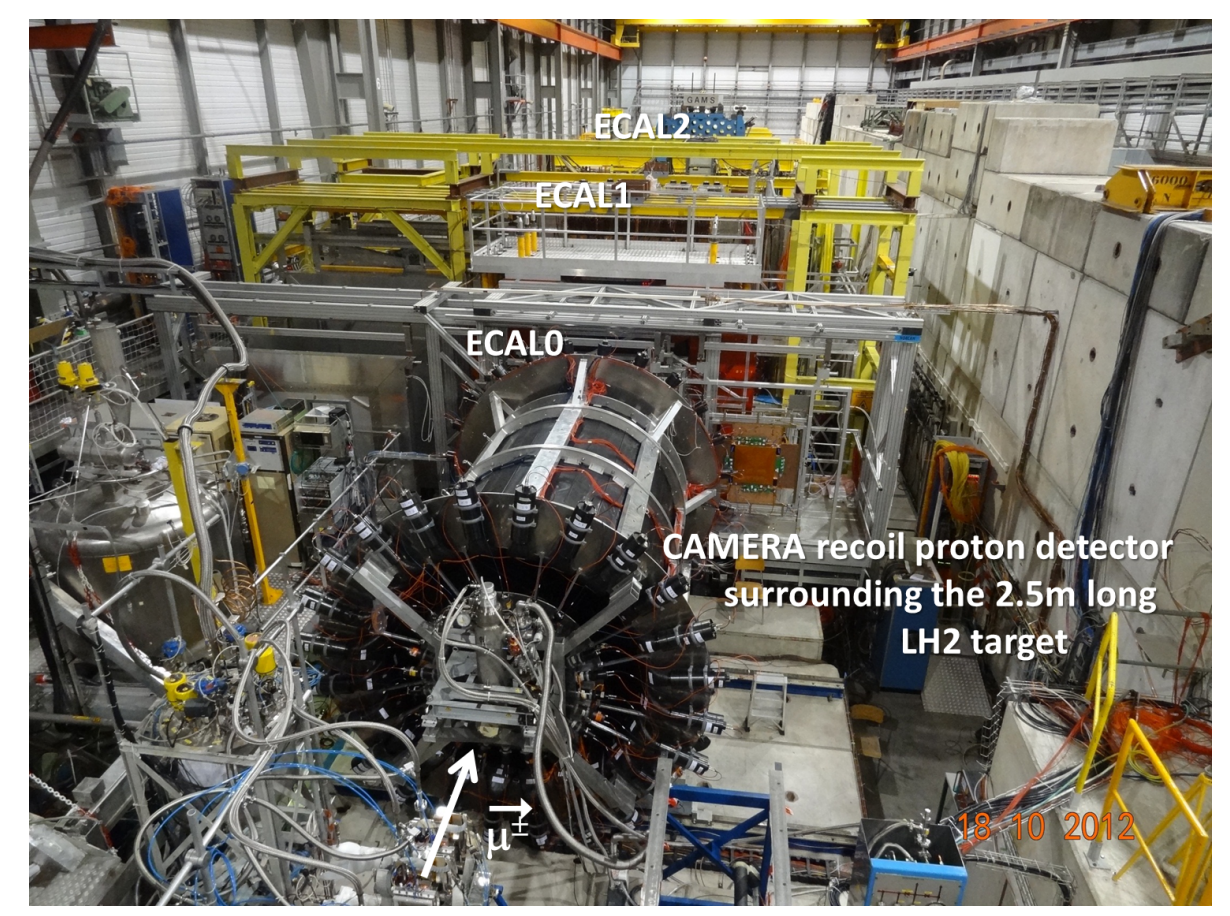

Figure 18. The COMPASS set-up at CERN in October 2012 with the recoil proton detector installed around a $2.5 \mathrm{~m} \mathrm{LH} 2$ target just before the calorimeter ECAL0 and the $60 \mathrm{~m}$ long COMPASS spectrometer.

$\cos \phi$ modulation of the asymmetry, integrated over $Q^{2}$ as a function of $t$ in 6 domains in $x_{B}$. This modulation is related to the real part of the CFF $\mathcal{H}$ which was found positive at HERA [35] and negative at HERMES [49]. The kinematic domain of COMPASS, in particular the region $0.005<x_{B}<0.03$ (see the 3 top panels of Fig.4) is expected to allow the determination of the $x_{B}$ position of the node of this function, which is an essential input for any global fitting procedure of GPDs.

Fig. 21 shows the imaginary part (top) and the real part (bottom) of the CFF $\mathcal{H}$ as a function of $x_{B}$ for 2 values of $t$
0 and $-0.3 \mathrm{GeV}^{2}$ as far as they are known from the present available results from HERA, HERMES and JLab. It exists two solutions at large $x_{B}$ including (blue line) or not (green line) results from Jlab Hall A. Whether this is a realistic scenario is an open problem which should be addressed soon thanks to the future new results from JLab 12 GeV. The COMPASS data will bring also important information on the exact position of the node for the real part (bottom) of the CFF. All these new results will improve considerably the global determination of GPDs in a large range of $x_{B}$ from sea quarks to valence quarks. A future 

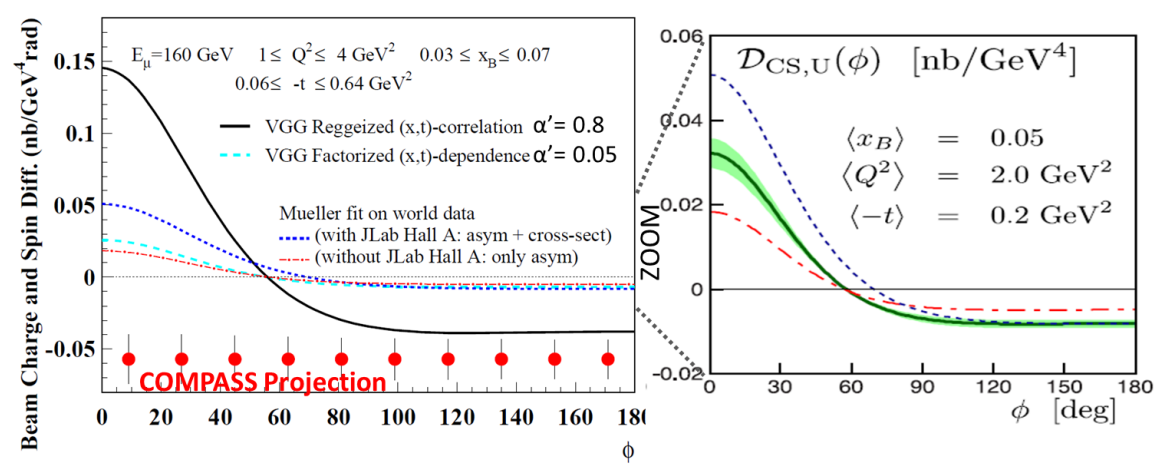

Figure 19. Projected statistical (error bars) accuracy for the measurement of the $\phi$ dependence of $\mathrm{d} \sigma^{\stackrel{+}{\leftarrow}}-\mathrm{d} \sigma^{\rightarrow}$ for $0.03 \leq x \leq 0.07$ and $1 \leq Q^{2} \leq 4 \mathrm{GeV}^{2}$ and for 280 days of running time with a $2.5 \mathrm{~m} \mathrm{LH}_{2}$ target, an intensity of $4.6 \times 10^{8} \mu$ in a $48 \mathrm{~s}$ SPS spill period and an overall global efficiency of $10 \% .3$ predictions are shown: VGG model $[9,13,14]$ (black and cyan curves on left), GK model [21] (green curve on right) and calculation from a global fit to present world data [18] with (blue curve) and without (red curve) including JLab Hall A data. Figure on left from ref. [69]. Figure on right from ref.[21].
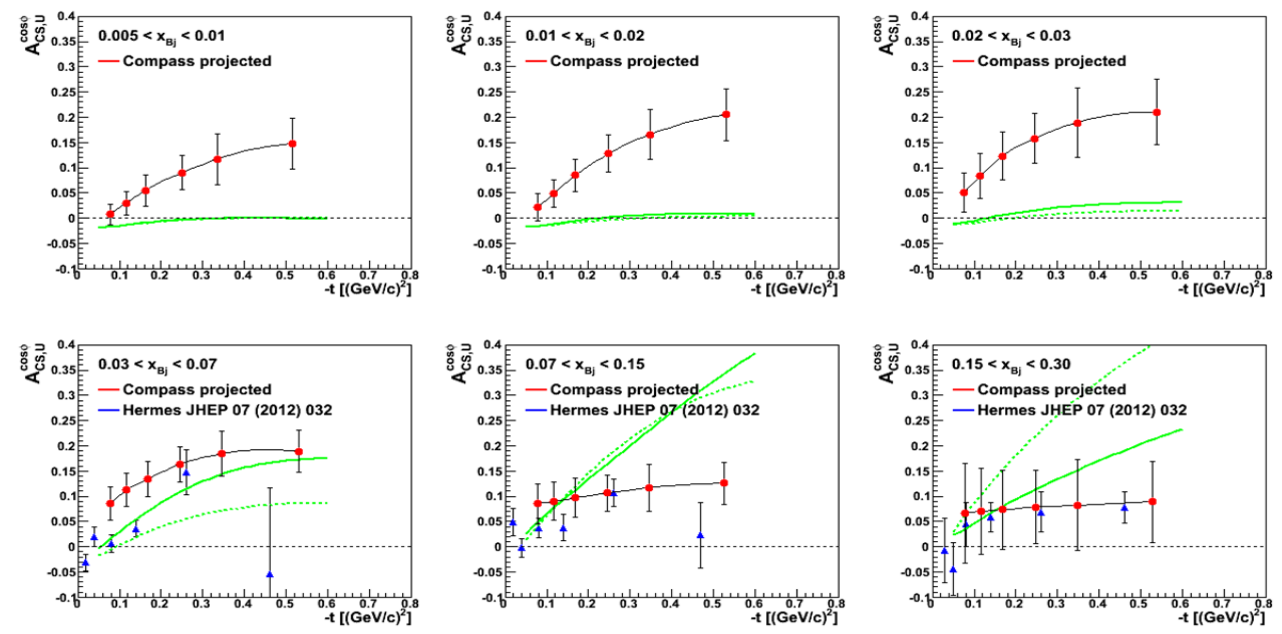

Figure 20. Projected statistical accuracy for the amplitude of the $\cos \phi$ modulation of the beam charge and spin asymmetry. Projections (red points) are calculated using the VGG model $[9,13,14]$. The green curves show latest calculations [18] based on a global fit on world data including JLab Hall A (solid line) or not (dotted line). The blue triangles at large $x_{B}$ show HERMES results [49].

collider of large energy will complete the description in the gluon sector.

\section{Hunting the GPD E through DVCS on the neutron or with transversely polarized target}

To access the GPD $E$ requires beam polarization dependence of DVCS using a neutron (or deuterium) target or asymmetry measurements for DVCS or exclusive vector meson production with transversely polarized target (see Eqs.(18) and (20)). This provides kinematics wise unsuppressed access to the GPD $E$. Production of $\rho$ mesons on a transversely polarized target at COMPASS [47] shows a very small contribution of the GPD $E$ but a significant contribution of the chiral-odd GPD $H_{T}$ (which has the transversity as TMD partner) in rather good agreement with the GK model. Earlier DVCS measurements on a neutron target at JLab [59] and on a transversely polar- ized target at HERMES [50] suffer from lack of precision. However attempts to extract information about quark total angular momentum have been performed by fitting the parameters $J_{u}$ and $J_{d}$ of the VGG model to the DVCS measurements (see Fig. 22). Although these analyses are very model dependent, the results agree surprisingly well with models and lattice expectations. Several challenging experiments at JLab [73, 74], at COMPASS [69] and with future colliders will have for goal to clarify this issue.

\section{References}

[1] S. Meissner, A. Metz, K. Goeke, Phys. Rev. D76, 034002 (2007); S. Meissner, A. Metz, M. Schlegel, JHEP 056, 0908 (2009).

[2] C. Lorcé, B. Pasquini, Phys. Rev. D84, 034039 (2011); C. Lorcé, B. Pasquini, M. Vanderhaeghen, JHEP 1105, 041 (2011).

[3] D. Müller at al., Fortschr. Phys.42, 101 (1994). 

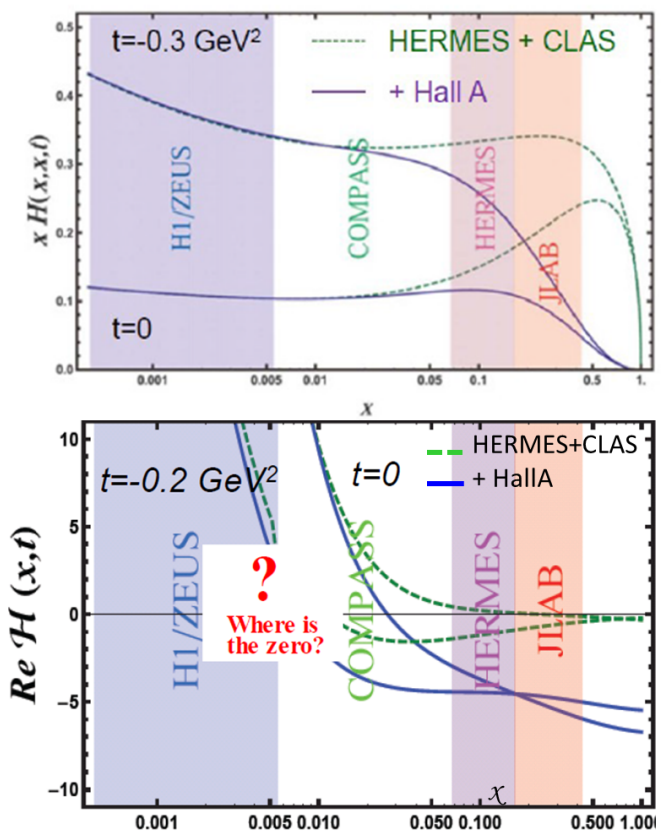

Figure 21. Imaginary part (top) and the real part (bottom) of the $\mathrm{CFF} \mathcal{H}$ as a function of $x_{B}$ for 2 values of $|t|$. Figure from Ref. [18].

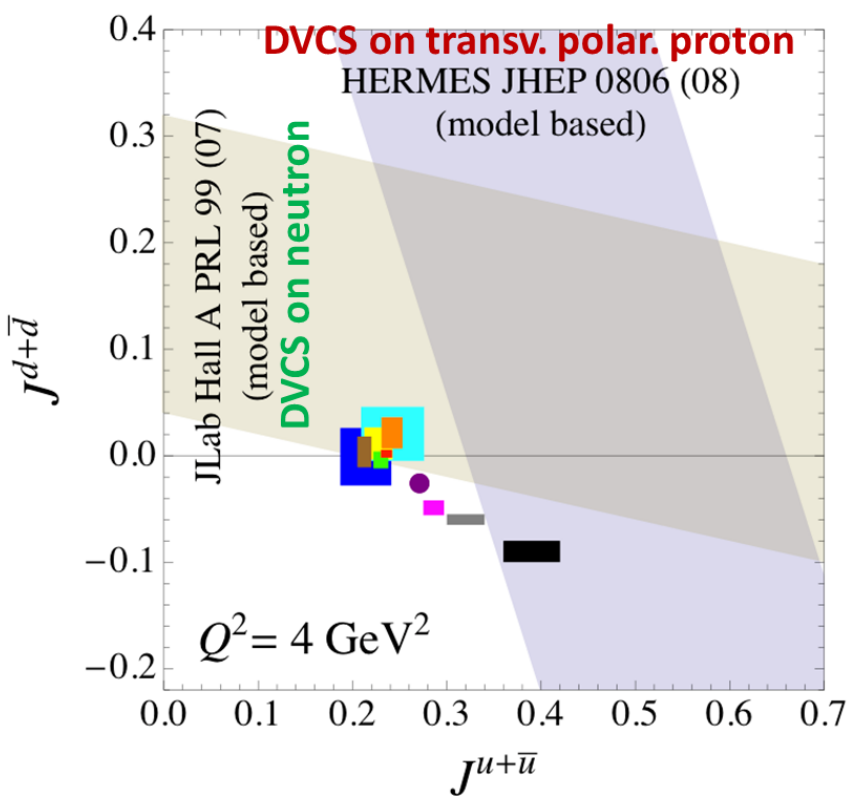

Figure 22. Jlab Hall A DVCS measurement from the neutron are interpreted to give $J_{d}+J_{u} / 5=0.18 \pm 0.14$ [59] whereas HERMES results from the transversely polarized proton give $J_{d}+$ $J_{u} / 5=0.18 \pm 0.14$ [59] using the same VGG model. Predictions from GPD models (GK [16] (cyan), VGG [9, 13, 14] (brown), Goldstein et al. [20] (rose)), form factor considerations (Diehl et al. [15] (violet)), semi-inclusive measurements (Bacchetta and Radici [83]), lattice predictions (LHPC [27] (blue), [28] (red) and QCDSF [29] (green)), quark models ( Wakamatsu [84] (yellow) and Thomas [85] (black and grey))
[4] A. V. Radyushkin, Phys. Lett. B385, 333 (1996); Phys. Rev. D56, 5524 (1997).

[5] X.-D. Ji, Phys. Rev. Lett. 78, 610 (1997); Phys. Rev. D55, 7114 (1997).

[6] J.C. Collins, L. Frankfurt, M. Strikman, Phys. Rev. D56, 2982 (1997).

[7] M. Polyakov, Phys. Lett. B555, 57 (2003).

[8] M. Burkardt, Phys. Rev. D62, 071503 (2000), Erratum-ibid. D66, 119903 (2002); Int. J. Mod. Phys. A18, 173 (2003); Phys. Lett. B595, 245 (2004).

[9] K. Goeke, M.V. Polyakov, M. Vanderhaeghen, Prog. Part. in Nucl. Phys. 47, (2001) 401.

[10] M. Diehl, Generalized Parton Distributions, Phys. Rept. 388, 41-277 (2003), DESY-thesis-2003-018, hep$\mathrm{ph} / 0307382$.

[11] A.V. Belitsky, A.V. Radyushkin, Phys. Rept. 418, 1 (2005).

[12] J.C. Collins, A. Freund, Phys. Rev. D59, 074009 (1999).

[13] M. Vanderhaeghen, P.A.M. Guichon, M. Guidal, Phys. Rev. Lett. 80 (1998) 5064; Phys. Rev. D60 094017 (1999).

[14] M. Guidal, M.V. Polyakov, A.V. Radyuskin, M. Vanderhaeghen, Phys. Rev. D72, 054013 (2005).

[15] M. Diehl, Th. Feldmann, R. Jakob, P. Kroll, Eur. Phys. J. C39, 1 (2005).

[16] S.V. Goloskokov, P. Kroll, Eur. Phys. J. C42, 281 (2005), Eur. Phys. J. C50, 829 (2007), Eur. Phys. J. C53, 367 (2008), Eur. Phys. J. C59, 809 (2009), Eur. Phys. J. C65, 137 (2010), Eur. Phys. J. A47, 112 (2011), Eur. Phys. J. C74, 2725 (2014).

[17] K. Kumericki, D. Müller, K. Passek-Kumericki, Nucl. Phys. B794, 244-323 (2008).

[18] K. Kumericki, D. Müller, Nucl. Phys. B841, 1-58 (2010).

[19] D. Müller, arXiv:1405.2817 (2014).

[20] G. R. Goldstein, J.O. Hernandez, S. Liuti, Phys. Rev. D84, 034007 (2011).

[21] P. Kroll, H. Moutarde, F. Sabatié, Eur. Phys. J. C73, 2278 (2013).

[22] M. Guidal, H. Moutarde, M. Vanderhaeghen, Rept. Prog. Phys. 76066202 (2013).

[23] V.M. Braun, A.N. Manashov, D. Mueller, B. Pirnay, Phys. Rev. D89, 074022 (2014).

[24] H. Moutarde, Phys. Rev. D79, 094021 (2009).

[25] M. Guidal, Phys. Lett. B689, 156 (2010).

[26] K. Kumericki, D. Müller, M. Murray, Phys. Part. Nucl. 45723 (2014).

[27] D. Brommel et al., QCDSF-UKQCD Collaborations, Pos LAT2007, 158 (2007) arXiv:0710.1534.

[28] M. Göckeler et al., QCDSF-UKQCD Collaborations, Phys. Rev. Lett. 98, 222001 (2007).

[29] P. Hägler et al., LHPC Collaboration, Phys. Rev. D77, 094502 (2008).

[30] J.D. Bratt et al., LHPC Collaboration, Phys. Rev. D82, 094502 (2010).

[31] H1, C. Adloff et al., Phys. Lett. B517, 47 (2001). 
[32] H1, A. Aktas et al., Eur. Phys. J. C44, 1 (2005).

[33] H1, F.D. Aaron et al., Phys. Lett. B659, 796 (2008).

[34] H1, F.D. Aaron et al., Phys. Lett. B681, 391 (2009).

[35] H1, L. Schoeffel et al., proceedings of the 15th International Workshop on Deep-Inelastic Scattering (DIS2007), arXiv:0705.2925.

[36] H1, S. Aid et al., Nucl. Phys. B463, 3 (1996); C. Adloff et al., Z. Phys. C75, 607 (1997); Eur. Phys. J. C13, 371 (2000); A. Aktas et al., Phys. Lett. B638, 422 (2006); F. Aaron et al., JHEP 1005, 032 (2010).

[37] H1, C. Adloff et al., Phys. Lett. B483, 360 (2000).

[38] H1, A. Aktas et al., Eur. Phys. J. C46, 585 (2006); Phys. Lett. B568, 205 (2003);

[39] H1, C. Adloff et al., Phys. Lett. B483, 23 (2000).

[40] ZEUS, S. Chekanov et al., Phys. Lett. B573, 46 (2003).

[41] ZEUS, S. Chekanov et al., JHEP 0905108 (2009).

[42] ZEUS, M. Derrick et al., Z. Phys. C73, 253 (1997); J. Breitweg et al., Eur. Phys. J. C2, 247 (1998); Eur. Phys. J. C6, 603 (1999); Eur. Phys. J. C14, 213 (2000); S. Chekanov et al., Eur. Phys. J. C26, 389 (2003); PMC Phys. A1, 6 (2007).

[43] ZEUS, M. Derrick et al., Z. Phys. C73, 73 (1996); J. Breitweg et al., Phys. Lett. B487, 273 (2000).

[44] ZEUS, M. Derrick et al., Phys. Lett. B377, 259 (1996); S. Chekanov et al., Nucl. Phys. B718, 3 (2005).

[45] ZEUS, S. Chekanov et al, Eur. Phys. J. C24 345 (2002).

[46] ZEUS, S. Chekanov et al, Nucl. Phys. B695 3 (2004).

[47] COMPASS, M. Alekseev et al., Eur. Phys. J. C52 255 (2007); C. Adolph et al., Nucl. Phys. B865, 1 (2012); Phys. Lett. B731, 96 (2014).

[48] HERMES, A. Airapetian et al., Phys. Rev. Lett. 87 (2001) 182001.

[49] HERMES, A. Airapetian et al., Phys. Rev. D75 011103 (2007).

[50] HERMES, A. Airapetian et al., JHEP 0806, 066 (2008); Phys. Lett. B704, 15 (2011).

[51] HERMES, A. Airapetian et al., JHEP 0911, 083 (2009).

[52] HERMES, A. Airapetian et al., JHEP 1006, 019 (2010).

[53] HERMES, A. Airapetian et al., JHEP 1207, 032 (2012).

[54] HERMES, A. Airapetian et al., JHEP 1210, 042 (2012).

[55] HERMES, A. Airapetian et al., Eur. Phys. J. C17, 389 (2000); Eur. Phys. J. C29, 171 (2003); Eur. Phys. J. C62, 659 (2009); Phys. Lett. B679, 100 (2009); Eur. Phys. J. C71, 1609 (2011).

[56] HERMES, A. Borissov et al., proceedings of DIFFRACTION2000, Cosenza, Italy (2000); DESYHERMES-00-055.
[57] HERMES, A. Airapetian et al., Phys. Lett. B659, 486 (2008); Phys. Lett. B682, 345 (2010).

[58] JLab 6 GeV, Hall A, C. Munoz Camacho et al., Phys. Rev. Lett. 97, 262002 (2006).

[59] JLab 6 GeV, Hall A, M. Mazouz et al., Phys. Rev. Lett. 99, 242501 (2007).

[60] JLab 6 GeV, CLAS, S. Stepanyan et al., Phys. Rev. Lett. 87182002 (2001).

[61] JLab 6 GeV, CLAS, S. Chen et al., Phys. Rev. Lett. 97, 072002 (2006).

[62] JLab 6 GeV, CLAS, F.X. Girod et al., Phys. Rev. Lett. 100, 162002 (2008).

[63] JLab 6 GeV, CLAS, G. Gavalian et al., Phys. Rev. C80, 035206 (2009).

[64] C. Hadjidakis et al., Phys. Lett. B605, 256 (2005); S.A. Morrow et al., Eur. Phys. J. A39, 5 (2009); R. De Massi et al., Phys. Rev. C77, 042201 (2008);

[65] L. Morand et al., Eur. Phys. J. A24, 445 (2005);

[66] J.P. Santoro et al., Phys. Rev. C78, 025210 (2008);

[67] E. Fuchey et al. Phys. Rev. C83, 025201 (2011); I. Bedlinskiy et al., Phys. Rev. Lett. 109, 0112001 (2012);

[68] H.P. Blok et al., Phys. Rev. C78, 045202 (2008);

[69] COMPASS-II proposal, CERN/SPSC-2010-014, SPSC-P-340, http://cdsweb.cern.ch/record/1265628/ files/SPSC-P-340.pdf, May 17, 2010.

[70] JLab12, Hall A, E12-06-114, DVCS on LH2 target

[71] JLab12, Hall C, E12-13-010, DVCS on LH2 target

[72] JLab12, CLAS, E12-06-119, DVCS on LH2 target (and long. pol.)

[73] JLab12, CLAS, E12-11-003, DVCS on neutron

[74] JLab12, CLAS, E12-12-010, DVCS on the HD ice target

[75] Dudek et al., Eur. Phys. J. A48, 187 (2012)

[76] EIC in USA, White paper, Electron Ion Collider: The QCD frontier. Understanding the glue that binds us all. BNL-98815-2012-JA, JLAB-PHY-12-1652

[77] E.C. Aschenauer, S. Fazio, K. Kumericki, D. Mueller, JHEP 1309093 (2013).

[78] EIC at HIAF in China, talk given by Xurong Chen, SPIN2014, Beijing

[79] H1, S. Aid et al., Nucl. Phys. B470, 3 (1996).

[80] M. Diehl, T. Gousset, B. Pire, J. Ralston, Phys. Lett. B411, 193 (1997).

[81] M. Diehl, contribution to the workshop "Future Physics @ COMPASS", 26-27 September 2002, CERN-2004-011.

[82] A.V. Belitsky, D. Müller, A. Kirchner, Nucl. Phys. B629, 323 (2002).

[83] A. Bacchetta, M. Radici, Phys.Rev.Lett. 107, 212001 (2011).

[84] M. Wakamatsu, Eur.Phys.J. A44, 297 (2010).

[85] A. W. Thomas, Phys. Rev. Lett. 101, 102003 (2008). 